\title{
Plastic and evolutionary responses of cell size and number to larval malnutrition in Drosophila melanogaster
}

\author{
R. K. VIJENDRAVARMA, S. NARASIMHA, T. J. KAWECKI
}

Department of Ecology and Evolution, University of Lausanne, Switzerland

Correspondence: Tadeusz J. Kawecki, Department of Ecology and Evolution, University of Lausanne, Biophore, CH 1015 Lausanne, Switzerland; email: tadeusz.kawecki@unil.ch

Journal of Evolutionary Biology 24: 897-903 (2011),

doi: 10.1111/j.1420-9101.2010.02225.x

The content of this preprint is identical with the published version except for a few inconsequential changes in the proofs

\begin{abstract}
Both development and evolution under chronic malnutrition lead to reduced adult size in Drosophila. We studied the contribution of changes in size versus number of epidermal cells to plastic and evolutionary reduction of wing size in response to poor larval food. We used flies from six populations selected for tolerance to larval malnutrition and from six unselected control populations, raised either under standard conditions or under larval malnutrition. In the control populations phenotypic plasticity of wing size was mediated by both cell size and cell number. In contrast, evolutionary change in wing size, which was only observed as a correlated response expressed on standard food, was mediated entirely by reduction in cell number. Plasticity of cell number had been lost in the selected populations, and cell number did not differ between the sexes despite males having smaller wings. Results of this and other experimental evolution studies are consistent with the hypothesis that alleles which increase body size through prolonged growth affect wing size mostly via cell number, while alleles which increase size through higher growth rate do so via cell size.
\end{abstract}

Keywords: experimental evolution, body size, cell size, cell number, nutritional stress, plasticity, imaginal disks, wing

\section{Introduction}

Body size influences multiple aspects of animal biology and is thus a target of natural selection (e.g., Blanckenhorn, 2000). Variation in body size has usually both a genetic and an environmental component; both evolution and plasticity of body size have been extensively studied. In contrast, less attention has been devoted to evolutionary and plastic changes in cell size and /or cell number which underlie changes in body size (Azevedo \& Leroi, 2001). Nonetheless, cell size and number may affect fitness independently of the size of the body and its parts and organs. For example, at least in vertebrates, interspecific variation in cell size is negatively correlated with metabolic rates per gram of tissue (Goniakowska, 1973, Vinogradov, 1997, Gregory, 2002, Starostova et al., 2009). Achieving the same size with a greater number of smaller cells implies more somatic mitotic divisions, leading to a greater risk of cancer and other tumours. This is well documented in mammals (Preston-Martin et al., 1990), but likely to apply more generally; e.g., in Drosophila larval tumours develop most readily in the highly mitotically active imaginal discs and brain (Hariharan \& Bilder, 2006, Beaucher et al., 2007). Across species within most taxa cell size is strongly positively correlated with genome size; thus, genome size may impose a limit on cell size (Arendt, 2007). However, it has also been suggested that evolution of genome size is partially driven by selection on cell size (Kozlowski et al., 2003). Thus, whether a change in body size involves changes in cell size versus cell number may affect correlated responses in ecologically relevant traits.

This paper addresses the contribution of cell size and number to plastic and evolutionary changes in Drosophila melanogaster wing size in response to larval malnutrition. Following other studies (Partridge et al., 1994a, French et al., 1998, Azevedo et al., 2002), we concentrate on the wing because it provides a convenient measure of structural body size, and because it is particularly amenable to estimating cell size and number. Some results suggest that plastic responses of wing blade cell size may be representative of epidermal cell size in other body parts, although this does not seem to hold for evolutionary changes (Azevedo et al., 2002). 
Several past studies addressed the cellular bases of plastic and evolutionary changes of Drosophila wing size (reviewed in Arendt 2007). As most invertebrates, when raised at lower temperatures Drosophila develop more slowly and eclose at a larger adult body size, both in terms of dry weight and wing size. Similarly, Drosophila populations reared at low temperature for multiple generations evolve to be genetically larger (e.g., Cavicchi et al., 1985; Huey et al., 1991; Partridge et al., 1994a). In D. melanogaster, both the phenotypic plasticity and the genetic adaptation of wing size to temperature under laboratory conditions are mediated mostly or entirely by changes in cell size, with some contribution of cell number to the plastic response in males (Cavicchi et al., 1985, Partridge et al., 1994a, French et al., 1998, Azevedo et al., 2002). In a thermal evolution experiment in D. subobscura evolutionary decrease in wing size was mediated mostly by cell size in females and mostly by cell number in males (Santos et al., 2005).

In contrast to thermal plasticity, the plastic reduction of wing size under nutritional stress and larval crowding has been mainly attributed to reduction in cell number (Robertson, 1959). DeMoed et al (1997) reports an interaction between the two factors: at $27.5^{\circ} \mathrm{C}$, plastic response of wing size to food was mediated by changes in cell number, while at $15^{\circ} \mathrm{C}$ it involved both cell size and cell number. Evolutionary response of cell size versus cell number to poor nutrition has not been investigated.

Also in contrast to adaptation to low temperature, the evolution of larger wing size in response to low humidity is based entirely on increased cell number (Kennington et al., 2003). Increased cell number has also mediated the response to direct artificial selection for large body size (thorax length), but in the same study the response to selection for small body size has been mostly mediated by reduction in cell size, with a small contribution of reduction in cell number (Partridge et al., 1999). In another study, the response to artificial selection (up and down) on wing length was largely mediated by cell number, with some contribution of cell size (Noach et al., 1997). Finally, of two independent natural latitudinal clines in wing size in $D$. melanogaster, one seems mostly based on cell size and the other mostly on cell number (Zwaan et al., 2000). Similar idiosyncrasy of cellular basis of independent wing size clines was reported in D. subobscura (Calboli et al., 2003). Thus, the cellular basis of plastic and evolutionary responses of body size seems to depend on the focal environmental factor and possibly other conditions. It has also been proposed that it may reflect historical contingency, assuming that it is the wing size rather than cell size or number that is the target of selection (Zwaan et al., 2000).

In an attempt to synthesize these results, Arendt (2007) proposed that, at least in flies, the cellular basis of differences in wing size depends on differences in growth rate. Where smaller adult size is a consequence of slower larval growth (as is the case under poor nutrition), the difference in size would mostly be mediated by cell number. In contrast, where slower growth is associated with larger adult size (owing to much longer growth phase, as it the case for flies raised at low temperatures), the difference would mostly be mediated by cell size. This generalization is supported by plastic responses to food and temperature and to some degree by genetically-based patterns of geographic variation (Arendt, 2007). The results of laboratory evolution experiments are less consistent with this prediction. For example, in contrast to the plastic response to temperature, flies maintained at low temperatures evolve larger size mediated by faster growth, yet, the difference in adult size is based on cell size (Partridge et al., 1994a). In general, it remains open to what extent the evolutionary responses in cell size or number parallel the plastic responses for factors other than temperature.

In this study we take advantage of replicate fly populations subject to 73 generations of experimental evolution under chronic larval malnutrition. During those 73 generations the larvae were reared on a very poor food, on which non-adapted flies take $70 \%$ longer to develop from egg to adult, show $20 \%$ lower viability, and emerge at half the body weight of flies maintained on standard food. Adaptation to malnutrition in these selected populations included the evolution of faster growth on poor food, smaller body size and faster development, the latter resulting at least in part from a cut-off on developmental period imposed during selection (Kolss et al., 2009). By comparing, in a $2 \times 2$ design, the selected populations and unselected controls under standard and poor food conditions we investigate the relative contribution of cell size and number to both plastic and evolutionary responses of wing size to nutritional stress. Based on our results and other studies, we also propose a hypothesis that links the cellular basis of wing size evolution with underlying changes in growth rate versus length of the growth phase. 


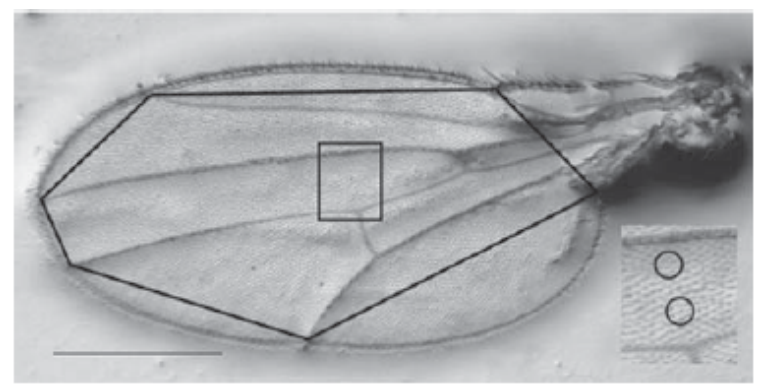

Figure 1: Drosophila wing; wing size was measured as the area of the polygon (scale bar $=0.5 \mathrm{~mm})$. Inset: the region opposite posterior cross-vein between $4^{\text {th }}$ and $5^{\text {th }}$ lateral vein (black rectangle), the circles indicated sampling areas used for estimating cell area.

\section{Material and Methods}

Six populations had been selected for tolerance to chronic larval malnutrition by being maintained on poor larval food for 73 generation; six control populations had been maintained on standard food. All were derived from the same base population, maintained for several years in the lab and traced to a single natural population (details in Kolss et al., 2009). The standard food contained $15 \mathrm{~g}$ agar, $30 \mathrm{~g}$ sucrose, $60 \mathrm{~g}$ glucose, $12.5 \mathrm{~g}$ dry yeast, $50 \mathrm{~g}$ cornmeal, $0.5 \mathrm{~g} \mathrm{MgSO}_{4}, 0.5 \mathrm{~g} \mathrm{CaCl}_{2}, 30 \mathrm{ml}$ ethanol, $6 \mathrm{ml}$ propionic acid, and $1 \mathrm{~g}$ nipagin per litre of water; the poor food contained $25 \%$ of those amounts of sugars, yeast and cornmeal. In the course of selection flies of both selection regimes were raised under a rather low density of 200 eggs per a vial containing $30 \mathrm{ml}$ of food. Thus, even under the poor food conditions the total energy content of the food was about $4.4 \mathrm{kcal}$, i.e., about $22 \mathrm{cal}$ per larva. This is about ten times the energy content of a large $(0.4$ mg dry weight) pre-pupation larva. Thus, the main challenge for the larvae was poor food quality rather than competition. The selected populations were bred from flies that eclosed first (within 14-17 days from oviposition depending on when a target of 200 adults was reached). Flies were raised at $25^{\circ} \mathrm{C}$ and $70 \%$ humidity.

Prior to the assays reported here all populations were reared for two generations on standard food. The flies thus raised were allowed to mate for two days and then allowed to oviposit overnight. Four $160 \mathrm{ml}$ vials, two with $30 \mathrm{ml}$ of standard food and two with $30 \mathrm{ml}$ of poor food were set-up per population, each seeded with 200 eggs, i.e., under the density and conditions used in the selection regimes. Wing measurements were done on five males and five females collected randomly fifteen days later and stored in 100\% ethanol. The design thus consisted of two main factors: past selection regime (selected vs. control) and current food (standard vs. poor), with replicate populations nested in selected regime, and two replicate vials per population $\times$ food combination.

The left wing of each fly was mounted on a glass slide in lactic acid/ethanol (6:5) (Trotta et al., 2007) and photographed at $40 \times$ magnification. The outline of each wing was traced to the nearest micrometer (figure 1) and the area of the resulting polygon was estimated using ImageJ (Abramoff et al., 2004). This area was treated as a proxy measure of wing size; we refer to it below as "wing area". Another image of the region between the $4^{\text {th }}$ and $5^{\text {th }}$ lateral vein opposite of the posterior crossvein was taken at $200 \times$ magnification. In this image, the trichomes with roots within two circular sampling areas of $1000 \mu \mathrm{m}^{2}$ each (figure 1) were counted (each epidermal cell produces one trichome). The mean cell area was estimated as $2000 \mu \mathrm{m}^{2} / \mathrm{number}$ of trichomes in the two areas.

To relate differences in wing size to differences in body weight, adult dry weight was measured in a separate experiment. Per population we set-up four vials with standard food and four with poor food, each seeded with 200 eggs. On emergence, twelve adults of each sex were collected at random from each vial, dried at $70^{\circ} \mathrm{C}$ for three days and then weighed as a group.

To obtain a relative estimate of total cell number in the wing, we divided the wing area by cell area. It should be noted that this number underestimates the total number of cells building the wing, first because our measure of wing area underestimates wing size (see above), and second, because cells building the veins are smaller. However, to the degree that changes in cell size are isometric, our estimates of cell number should be proportional to the actual cell number in the entire wing. The wing area, cell area, cell number and weight data were $\log$ transformed for the analysis. We calculated the mean values of the three wing measures (log wing area, log cell area and log cell number) for each of the two vials per population. Similarly, mean log adult dry weight of each sex was estimated for each of the four vials per population and food treatment. 
These values were subject to an analysis of variance (using JMP 7.0 statistical software); selection regime, food and sex were treated as fixed factors, the replicate populations (nested within selection regimes) and interaction between populations and the fixed factors were treated as a random factors. The analysis thus treats the populations as the level or replication relevant to testing hypotheses about the effects of selection regime, food treatment and sex. We also analyzed the results separately for each food treatment. The F-tests for the fixed factors resulting from these analyses are summarized in Table 1 .

\section{Results}

The results for dry body weight confirmed the pattern observed in a previous assay (Kolss et al., 2009). Flies raised on poor food were 40-55 \% lighter (figure 2A). Selected populations had evolved a lower body weight than control populations, but this reduction was much more pronounced on the standard than on the poor food. When tested separately, on the poor food the difference between selected and control populations was only marginally significant in the present study $(P=$ $0.054)$, although this difference was significant in a previous assay (Kolss et al., 2009). The pattern of body weight differences was similar between the sexes, although the relative magnitude of the plastic response was somewhat greater in females.

The plastic reduction of body weight in flies raised on poor food was associated with reduction in wing area; as for body weight, this response tended to be more pronounced in females than males ( 22 vs. $17 \%$ for control populations, Figure 2B). Both sets of populations showed similar plasticity in cell size (Figure 2C); although it was more pronounced in females, it was also significant in males $\left(F_{1,10}=20.7\right.$, $P=0.0011)$. However, control populations also showed pronounced plasticity in cell number $\left(F_{1,5}=\right.$ $15.7, P=0.011)$ while this plasticity was absent in the selected populations $\left(F_{1,5}=0.2, P=0.65\right.$, note the regime $\times$ food interaction in table 1$)$. Rather, the plasticity of wing area in the selected populations was mediated entirely by cell size.
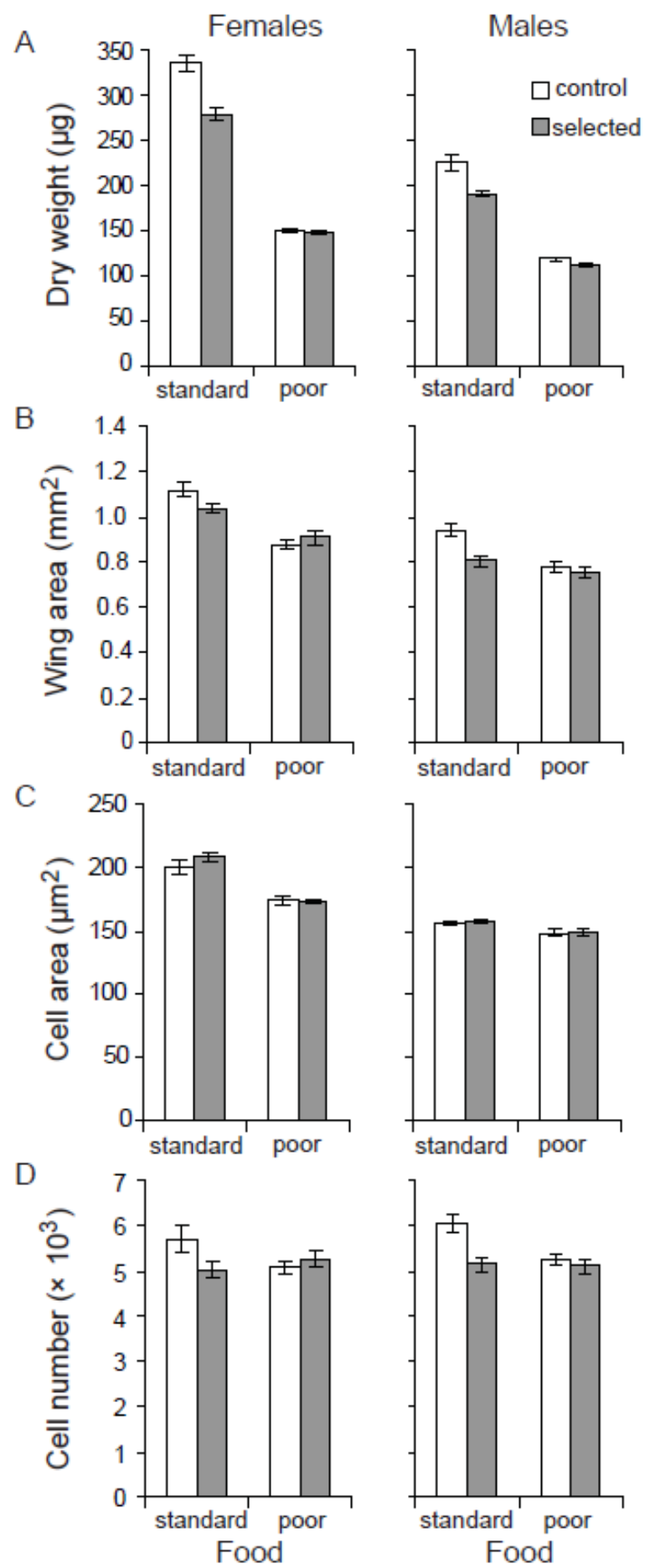

Figure 2: Means ( \pm S.E) of the traits of flies from the control and selected populations raised on poor and standard food.

This change in plasticity can be understood by analyzing the evolutionary responses. The selection regime did not consistently affect wing area, cell size and cell number expressed by flies raised on the poor food. However, when raised on the standard food, the selected flies of both sexes had smaller wings than controls (Figure 2B). In contrast to the plastic response, this correlated evolutionary response to selection for tolerance to poor food was mediated entirely by reduction in cell number. As a result, irrespective of the food treatment, the wings of selected flies had as few cells as those of the control flies raised on the poor food (Figure 2D). Sexual dimorphism in wing size was entirely mediated by cell size; despite having smaller 
wings, males had as many wing cells as females (figure 2C,D). Variation among the replicate populations did not affect any of the traits consistently across food types (all $P>0.4$, not reported in Table 1). We detected an interaction between population and food type for dry weight $(P<0.01)$; when analyzed separately, population affected dry weight on the standard food $(P<0.001)$. We also found interaction between population and sex for wing area and cell number on standard food (both $P<0.001$ ), food $\times \operatorname{sex} \times$ population interaction in the joint analysis of wing area $(P=0.02)$. Other interaction terms including population were not significant.

\section{Discussion}

Our results indicate that the plastic response of the control populations to poor larval nutrition involves a reduction in wing size, mediated by reduction in both cell number and, particularly in females, cell size. This plastic response is likely to be mediated, at least in part, by insulin signalling, which plays a central role in regulating growth responses to nutrition in Drosophila larvae. In parallel to the plastic response to our poor food (Kolss et al., 2009), mutants with reduced insulin signalling grow slowly, take long to develop, emerge at reduced adult size and with high proportional lipid content (Chen et al., 1996, Bohni et al., 1999). Insulin signalling promotes both cell proliferation and cell growth (Wu \& Brown, 2006). However, cell proliferation within wing imaginal discs is to some degree regulated autonomously (Neto-Silva et al., 2009), and is not affected by mild reduction in insulin signalling, which only reduces wing cell size (Shingleton et al., 2005). More severe reduction in insulin signalling also reduces cell proliferation without further reduction in cell size, so that mutants with strongly reduced insulin signalling have both fewer and smaller cells (Shingleton et al., 2005). The body mass of our flies raised on the poor food is as low as that of those mutants (Chen et al., 1996, Bohni et al., 1999). Thus, the contribution of both cell number and cell size to the plastic response of the control flies is consistent with the hypothesis of this response being regulated by a strong reduction in insulin signalling.

The large contribution of cell size to the plastic response contradicts Arendt's (2007) prediction that, in cases where small size results from slow growth, the response should be mostly based on cell number, a result observed in earlier studies of wing size plasticity in response to food quality (Robertson, 1959, DeMoed et al., 1997). One possible reason could be the different ways of applying nutritional stress: those studies reduced the amount of protein (yeast) available to the larvae whereas in our study the protein and carbohydrate content of the food was reduced by the same factor. One can thus speculate that deficiency of protein versus sugar may affect cell growth and proliferation differently. This would be somewhat analogous to the finding that reduction of protein versus carbohydrate has different effects on adult lifespan (Mair et al., 2005). While it is protein that is more limiting for growth, major mechanisms regulating cell growth and proliferation, such as insulin and TOR signalling, respond mostly to sugar levels (reviewed in Edgar, 2006). Nonetheless, there could be other reasons for the discrepancy between our results and those of Robertson (1959) and DeMoed et al. (1997), such as an interaction of the nutrition treatment with fly genotype or laboratory conditions (Ackermann et al., 2001).

In addition to substantially improved viability and faster development, the evolutionary adaptation of the selected populations to the poor food conditions involved a small reduction in dry body weight (12\% in Kolss et al., 2009; $4 \%$ in this study). Nonetheless, we could not detect any associated reduction in wing area or the underlying cell size or cell number in selected flies raised on the poor food. However, as a correlated response to selection, the selected flies evolved considerably lower weight and smaller wing area than the control flies when raised standard food. This correlated response likely results from selection for fast development and low critical size at which pupation is initiated on the poor food (Kolss et al., 2009). In contrast to the plastic response, the evolutionary response of wing size has been mediated entirely by reduction in cell number. The cell number expressed on the standard food was reduced in the selected populations to the same value as the cell number expressed on the poor food by both selected and control populations. As a consequence, the plastic response of cell number has been lost; the plastic response of wing size in the selected populations seems based entirely on cell size. Thus, the superficial similarity of plastic and evolutionary responses to malnutrition masks differences in cellular basis, and thus presumably in the underlying mechanism.

The mechanism of the evolutionary response is unlikely to be based on diminished insulin signalling, also because low insulin signalling typically leads to prolonged development (Chen et al., 1996, Bohni et al., 
1999), whereas the selected flies evolved substantially faster development (Kolss et al., 2009). In holometabolous insects initiation of metamorphosis is triggered by reaching a critical size as assessed by the ring gland, leading to a cascade of hormonal changes which eventually cause the larva to stop feeding; the length of post-critical feeding period is insensitive to insulin signalling (Mirth et al., 2005, Edgar, 2006). This faster development means a shorter feeding phase, which seems largely responsible for the smaller size of selected flies on standard food. The cells of the wing imaginal discs proliferate intensely from the first larval instar until the critical size for pupation is reached early in the third (last) larval instar; afterwards the cells divisions continue at a slower rate under autonomous control until one day after pupation (Neto-Silva et al., 2009).

These considerations suggest that whether an evolutionary change in size will involve cell size versus cell number may depend on whether the change is mediated by the larval growth rate versus the length of the growth period. As summarized above, these two basic ways of determining adult size are modulated by different physiological mechanisms, and thus are likely to be affected by variation at different loci. Genetic variation that affects the length of growth period (by affecting the critical size) will be characterized by positive correlation between adult size and developmental time: genetically larger individuals will take more time to grow, leaving them more time for cell proliferation. Hence the prediction that, where evolution of larger body size is associated with prolonged development, the size differences should be mostly mediated by cell number. In contrast, genetic variation in growth rate implies a negative genetic correlation between adult size and developmental time. Faster-growing individuals will reach critical size earlier, and thus will have less time for cell proliferation in the imaginal disks. This could be partially or wholly compensated by insulin signalling-mediated increase in the rate of proliferation (Neto-Silva et al., 2009). However, the length of subsequent post-critical feeding period is not affected by growth rate (Edgar, 2006), and it is at this stage that differences in final weight will be produced under this scenario. Hence, we predict that evolved differences in body size which are negatively correlated with developmental time will be mostly mediated by cell size; the larger genotype may even have fewer cells.

We thus propose that the lower cell number of the selected populations reflects less time available for cell proliferation. This putative link between the cellular basis of wing size evolution and the size-developmental time relationship is supported by results of other evolutionary experiments. In particular, in Partridge et al. $(1994 a, b)$ the laboratory evolution of larger size under low temperature was associated with faster development and was mediated entirely by cell size; in fact, the larger cold-evolved flies tended to have fewer cells as predicted above. (A similar pattern was observed by Cavicchi et al. 1985, but the data on developmental time were not reported.) In contrast, evolution of large size driven by low humidity was mediated by cell number (Kennington et al. 2003); although that study did not report the developmental time, in other studies evolution of large size driven by low humidity was associated with prolonged development (Chippindale et al. 1998; Telonis-Scott et al., 2006). Similarly, selection for large thorax size has produced flies with prolonged development and large wings based entirely on cell number (Partridge et al., 1999). Finally, populations selected for small thorax evolved smaller size with no change in developmental time; they had both smaller cells and fewer cells (Partridge et al., 1999). However, the link between cell size and developmental time is not supported by a study in which flies were directly selected for small wing cell number or small wing cell size: neither selection regime produced a consistent correlated response in developmental time (Trotta et al., 2007). It also unclear whether geographical patterns in cell size and number are consistent with the above hypothesis. The two latitudinal clines in body size whose cellular basis has been studied by Zwaan et al (2000) do not show corresponding clines in developmental time (James et al., 1997; van 't Land et al. 1999). Developmental time for the D. subobscura clines studied by Calboli et al. (2003) has apparently not been reported.

It is plausible that in the long term any association between developmental time and the cellular basis of wing size evolution could be broken if there were selection on cell size acting separately from selection on total wing or body size. Nonetheless, the results of experimental evolution studies, including these reported here, are consistent with the hypothesis that alleles which increase size by prolonging growth affect size mostly via cell number, while alleles which affect size by increasing growth rate do so via cell size. If so, cellular basis of genetic differences in body size may be more often explained by the mechanism responsible for the body size difference than by selection on cell size or number or historical contingency. 


\section{Acknowledgements}

This work has been supported by the Swiss National Science Foundation. We thank the Cellular Imaging Facility of the University of Lausanne and A. Paradis for technical help, and anonymous reviewers for comments on an earlier version.

\section{References}

Abramoff, M. D., Magelhaes, P. J. \& Ram, S. J. 2004. Image Processing with ImageJ. Biophotonics International 11: $36-42$.

Ackermann, M., Bijlsma, R., James, A. C., Partridge, L., Zwaan, B. J. \& Stearns, S. C. 2001. Effects of assay conditions in life history experiments with Drosophila melanogaster. J. Evol. Biol. 14: 199-209.

Arendt, J. 2007. Ecological correlates of body size in relation to cell size and cell number: patterns in flies, fish, fruits and foliage. Biological Reviews 82: 241-256.

Azevedo, R. B. R., French, V. \& Partridge, L. 2002. Temperature modulates epidermal cell size in Drosophila melanogaster. J. Insect Physiol. 48: 231-237.

Azevedo, R. B. R. \& Leroi, A. M. 2001. A power law for cells. Proc. Natl. Acad. Sci. USA 98: 5699-5704.

Beaucher, M., Goodliffe, J., Hersperger, E., Trunova, S., Frydman, H. \& Shearn, A. 2007. Drosophila brain tumor metastases express both neuronal and glial cell type markers. Developmental Biology 301: 287-297.

Blanckenhorn, W. U. 2000. The evolution of body size: What keeps organisms small? Quart. Rev. Biol. 75: 385-407.

Bohni, R., Riesgo-Escovar, J., Oldham, S., Brogiolo, W., Stocker, H., Andruss, B. F., Beckingham, K. \& Hafen, E. 1999. Autonomous control of cell and organ size by CHICO, a Drosophila homolog of vertebrate IRS1-4. Cell 97: 865-875.

Calboli, F. C. F., Gilchrist, G. W. \& Partridge, L. 2003. Different cell size and cell number contribution in two newly established and one ancient body size cline of Drosophila subobscura. Evolution 57: 566-573.

Cavicchi, S., Guerra, D., Giorgi, G. \& Pezzoli, C. 1985. Temperature-regulated divergence in experimental populations of Drosophila melanogaster. I. Genetic and developmental basis of wing size and shape variation. Genetics 109: 665689.

Chen, C., Jack, J. \& Garofalo, R. S. 1996. The Drosophila insulin receptor is required for normal growth. Endocrinology 137: 846-856.

Chippindale, A. K., Gibbs, A. G., Sheik, M., Yee, K. J., Djawdan, M., Bradley, T. J. \& Rose, M. R. 1998. Resource acquisition and the evolution of stress resistance in Drosophila melanogaster. Evolution 52: 1342-1352.

DeMoed, G. H., DeJong, G. \& Scharloo, W. 1997. Environmental effects on body size variation in Drosophila melanogaster and its cellular basis. Genet. Res. 70: 35-43.

Edgar, B. A. 2006. How flies get their size: genetics meets physiology. Nature Reviews Genetics 7: 907-916.

French, V., Feast, M. \& Partridge, L. 1998. Body size and cell size in Drosophila: the developmental response to temperature. J. Insect Physiol. 44: 1081-1089.

Goniakowska, L. 1973. Metabolism, resistance to hypotonic solutions, and ultrastructure of erythrocytes of 5 amphibian species. Acta Biol. Cracov. Ser. Zool. 16: 114-134.

Gregory, T. R. 2002. A bird's-eye view of the C-value enigma: Genome size, cell size, and metabolic rate in the class aves. Evolution 56: 121-130.

Hariharan, I. K. \& Bilder, D. 2006. Regulation of imaginal disc growth by tumor-suppressor genes in Drosophila. Annu. Rev. Genet. 40: 335-361.

Huey, R. B., Partridge, L. \& Fowler, K. 1991. Thermal sensitivity of Drosophila melanogaster responds rapidly to laboratory natural selection. Evolution 45: 751-756.

James, A. C., Azevedo, R. B. R. \& Partridge, L. 1997. Genetic and environmental responses to temperature of Drosophila melanogaster from a latitudinal cline. Genetics 146: 881-890.

Kennington, W. J., Killeen, J. R., Goldstein, D. B. \& Partridge, L. 2003. Rapid laboratory evolution of adult wing area in Drosophila melanogaster in response to humidity. Evolution 57: 932-936.

Kolss, M., Vijendravarma, R. K., Schwaller, G. \& Kawecki, T. J. 2009. Life-history consequences of adaptation to larval nutritional stress in Drosophila. Evolution 63: 2389-2401.

Kozlowski, J., Konarzewski, M. \& Gawelczyk, A. T. 2003. Cell size as a link between noncoding DNA and metabolic rate scaling. Proc. Natl. Acad. Sci. USA 100: 14080-14085.

Mair, W., Piper, M. D. W. \& Partridge, L. 2005. Calories do not explain extension of life span by dietary restriction in Drosophila. Plos Biology 3: 1305-1311.

Mirth, C., Truman, J. W. \& Riddiford, L. M. 2005. The role of the prothoracic gland in determining critical weight for metamorphosis in Drosophila melanogaster. Current Biology 15: 1796-1807.

Neto-Silva, R. M., Wells, B. S. \& Johnston, L. A. 2009. Mechanisms of growth and homeostasis in the Drosophila wing. Annu. Rev. Cell Dev. Biol. 25: 197-220.

Noach, E. J. K., DeJong, G. \& Scharloo, W. 1997. Phenotypic plasticity of wings in selection lines of Drosophila melanogaster. Heredity 79: 1-9.

Partridge, L., Barrie, B., Fowler, K. \& French, V. 1994a. Evolution and development of body-size and cell-size in Drosophila melanogaster in response to temperature. Evolution 48: 1269-1276. 
Partridge, L., Barrie, B., Fowler, K. \& French, V. 1994b. Thermal evolution of pre-adult life-history traits in Drosophila melanogaster. J. Evol. Biol. 7: 645-663.

Partridge, L., Langelan, R., Fowler, K., Zwaan, B. \& French, V. 1999. Correlated responses to selection on body size in Drosophila melanogaster. Genet. Res. 74: 43-54.

Preston-Martin, S., Pike, M. C., Ross, R. K., Jones, P. A. \& Henderson, B. E. 1990. Increased cell division as a cause of human cancer. Cancer Res. 50: 7415-7421.

Robertson, F. W. 1959. Studies in quantitative inheritance. 12 Cell size and number in relation to genetic and environmental variation of body size in Drosophila. Genetics 44: 869-896.

Santos, M., Cespedes, W., Balanya, J., Trotta, V., Calboli, F. C. F., Fontdevila, A. \& Serra, L. 2005. Temperaturerelated genetic changes in laboratory populations of Drosophila subobscura: Evidence against simple climatic-based explanations for latitudinal clines. Am. Nat. 165: 258-273.

Shingleton, A. W., Das, J., Vinicius, L. \& Stern, D. L. 2005. The temporal requirements for insulin signalling during development in Drosophila. Plos Biology 3: 1607-1617.

Starostova, Z., Kubicka, L., Konarzewski, M., Kozlowski, J. \& Kratochvil, L. 2009. Cell size but not genome size affects scaling of metabolic rate in eyelid geckos. Am. Nat. 174: E100-E105.

Telonis-Scott, M., Guthridge, K. M. \& Hoffmann, A. A. 2006. A new set of laboratory-selected Drosophila melanogaster lines for the analysis of desiccation resistance: response to selection, physiology and correlated responses. J. Exp. Biol. 209: 1837-1847.

Trotta, V., Calboli, F. C., Ziosi, M. \& Cavicchi, S. 2007. Fitness variation in response to artificial selection for reduced cell area, cell number and wing area in natural populations of Drosophila melanogaster. BMC Evolutionary Biology 7: S10.

Van 't Land, J., van Putten, P., Zwaan, B., Kamping, A. \& van Delden, W. 1999. Latitudinal variation in wild populations of Drosophila melanogaster: heritabilities and reaction norms. J. Evol. Biol. 12: 222-232.

Vinogradov, A. E. 1997. Nucleotypic effect in homeotherms: Body-mass independent resting metabolic rate of passerine birds is related to genome size. Evolution 51: 220-225.

Wu, Q. \& Brown, M. R. 2006. Signalling and function of insulin-like peptides in insects. Annu. Rev. Entomol. 51: 1-24.

Zwaan, B. J., Azevedo, R. B. R., James, A. C., Van 'T Land, J. \& Partridge, L. 2000. Cellular basis of wing size variation in Drosophila melanogaster: a comparison of latitudinal clines on two continents. Heredity 84: 338-347.

Table 1: Summary of analysis of variance ( $F$-statistic and its significance) on the four traits, analyzed jointly on both food types, and separately on each food type. For all $F$-tests $d f=1,10$; population is a random factor nested within selection regime; tests for population and its interactions are not reported (see Results).

\begin{tabular}{|c|c|c|c|c|c|}
\hline Factor & Denominator MS & Dry weight & Wing area & Cell area & Cell number \\
\hline \multicolumn{6}{|l|}{ Both food types } \\
\hline regime & population & $27.7^{\star \star \star}$ & $7.1^{*}$ & 0.62 & $5.0^{*}$ \\
\hline food & food $\times$ population & $677.0^{\star \star \star}$ & $33.1^{\star \star *}$ & $100.1^{\star \star \star}$ & $5.6^{*}$ \\
\hline $\operatorname{sex}$ & sex $\times$ population & $1530.4^{\star \star \star}$ & $119.5^{\star \star \star}$ & $269.1^{\star \star \star}$ & 0.9 \\
\hline regime $\times$ food & food $\times$ population & $18.5^{\star \star}$ & $4.5 \dagger$ & 1.31 & $9.4^{*}$ \\
\hline regime $\times$ sex & sex $\times$ population & $7.8^{*}$ & $4.2 \dagger$ & 0.20 & 1.3 \\
\hline food $x \operatorname{sex}$ & food $\times$ sex $\times$ population & $412.4^{\star \star \star}$ & $3.9 \dagger$ & $29.5^{\star \star \star}$ & 1.6 \\
\hline regime $x$ food $x$ sex & food $\times$ sex $\times$ population & $16.9^{\star *}$ & 0.0 & 0.6 & 0.1 \\
\hline \multicolumn{6}{|l|}{ Standard food } \\
\hline sex & sex $\times$ population & $1244.5^{\star \star \star}$ & $75.4^{\star \star *}$ & $253.8^{\star \star \star}$ & 1.6 \\
\hline regime*sex & sex $\times$ population & $16.5^{\star \star}$ & 2.3 & 0.6 & 0.5 \\
\hline \multicolumn{6}{|l|}{ Poor food } \\
\hline regime & population & $4.1 \dagger$ & 0.0 & 0.00 & 0.0 \\
\hline sex & sex $\times$ population & $354.8^{\star \star \star}$ & $49.0^{\star \star *}$ & $98.0^{\star \star \star}$ & 0.0 \\
\hline regime ${ }^{\star}$ sex & sex $\times$ population & 1.2 & 2.1 & 0.0 & 1.8 \\
\hline
\end{tabular}

$\dagger \mathrm{P}<0.1,{ }^{*} \mathrm{P}<0.05,{ }^{* *} \mathrm{P}<0.01, * * * \mathrm{P}<0.001 ;$ all the remaining $\mathrm{P}>0.1$ 